\title{
Troubleshooting Payment Journey In Multilayer System Using Blockchain Technology
}

\author{
Ahmed Abd Elwasaa \\ Faculty of Computers and Information, \\ Menofia University, Egypt \\ ahmedabdelwasaa@outlook.com
}

\author{
Eman M. Mohamed \\ Faculty of Computers and Information, \\ Menofia University, Egypt \\ eman.mohamed@ci.menofia.edu.eg
}

\author{
Arabi Keshk \\ Faculty of Computers and Information, \\ Menofia University, Egypt \\ arabi.keshk@ci.menofia.edu.eg
}

\begin{abstract}
Blockchain technology is the most promising field in the recent decade and future in many different industries. It is based on storing data in a decentralized distributed database with an encrypted way. Permission blockchains are the most of interest to companies, organizations, and other agencies to maintain their internal processes because of their trust, high privacy and control. Nowadays we are living in digital transformation age that everything should be maintained and operated by a system to ease our daily tasks. Electronic payment systems are one of many shapes for digital transformation, and they have many challenges in sending or receiving transactions as time latency, scalability, performance, and throughput rate also there is a need to maintain any failure through the payment process till it is completed. Tracing failed transactions may take more time and effort to discover what is the root cause of failure and which component has affected it. Therefore, the proposed framework is suggested using private blockchain to overcome the time delay of tracking failures and improve performance and throughput. Our results gained from the proposed framework enables us from tracing the failed transactions with little consumed time which provides more performance and efficiency. Also, by increasing the performance of finding trouble.
\end{abstract}

Keywords-Blockchain, Electronic payment, Digital transformation, Fintech

\section{INTRODUCTION}

Blockchain is a shared distributed ledger that allows participants in various places to trade assets with each other safely and efficient way. As a system of records which it keeps transactions among participants in an immutable way in addition to identical copies available to all participants and what we will do by this a system, it will describe the purpose of blockchain such as bitcoin for example [1][13]. Blockchain also defines as a system of proof that has a smart contract which it implements the business logic and business rules through it in addition to the way of reading and writes in the ledger and for transactions that transmitted between participants, and they use the same smart contract, this ensures that everything happened in the same way and the same business logic and rules, blockchain achieves the consensus, which is a consensus process that applied at the level of a business network to check and verify with every transaction happened in blockchain network through all participants, or some of them who have access to the network. There are two broad categories of blockchain [1][18]: Permissioned networks and Permissionless networks. Permissioned network is a private blockchain network and everyone in this network is known Identity, it requires permission to join and participate the consensus. Banks, companies or other organizations can't afford to make their processes or data public, and this is where private blockchain network is too useful and they need to secure their data. There are many characteristics of permissioned blockchain including the following:

- Semi-Decentralized: in a private blockchain there is a govern and control by one organization and this decrease decentralize level because there is a central authority that approves who will be joining the network. The level of decentralization in a permissioned network depends on the choice of consensus algorithms that will be used in the blockchain network.

- Control and Governance: permissioned network is governing by one organization, and this organization can decide to include the validator nodes to validate and verify transactions.

- Customization: organization can customize their business network based on their needs.

- Efficient: private blockchain network is efficient when it comes to transaction speed, throughput, and scalability.

- Trust and Immutability: participants in a permissioned network trust in the organization as the centralized node knows about participants when they join the network, so it is partial immutability.

So permissioned network is most recommended for banking systems and electronic payment systems [1-2][21]. In another category, which is a permissionless network, it is a public network, so anyone can join the network, it requires no permission to join and interact with the network. The Participants can interact with blockchain network by assessing the transaction's validation or transmit the transaction to another participant in the network. There are many characteristics of permissionless blockchain include the following:

- Fully Decentralized: in the public network there is no central control, and anyone can access the network anytime he wants there is no permission at all.

- Anonymously and transparent: the public network is open for everyone, and every participant can see all transactions that created over the network.

- Immutability: all transaction that made over the network can't be modified.

A Public blockchain is most recommended for voting systems and cryptocurrency systems [18]. So, in this case 
study it is obvious that a permissioned network is the most suitable choice for the proposed framework as there is need to trace failed payment transactions through many components that payment journey crosses them to be completely succussed.

The rest of the paper is organized as follows: Section II gives background for blockchain and electronic payment, Section III gives the proposed framework in detail, Section IV discusses the evaluation result of the proposed framework, and conclude this paper in Section IIV.

\section{BACKGROUND}

\section{A. Blockchain}

Blockchain is distributed ledgers or databases running on many machines and anyone can access it. It is a unique type of computerized ledger with cryptographic methods and consensus to save and secure the data. It is designed to operate and run by a computer, where not just information can be shared or transmitted, but anything that has a value like money, music, intellectual property, which are called assets can be stored and traded securely. Trust is the important thing blockchain can provide where there are no intermediaries like banks or third parties, this idea ensures integrity and trust between participants. According to the internet was the first native digital medium for information, blockchain can be consider as a first native digital medium for value and has a big effect on the business and organizations [2-5]. the public ledger contains a chain of blocks where all verified transactions can be recorded. Each block is linked with a previous block, and the hash number is generated through mathematical calculations by participants in the business network. In order to protect this ledger, there are many encryption algorithms and consensus to prevent any modification of verified transactions and protect the blockchain from multiple attacks. Each transaction is hashed, and each block contains a set of transactions based on the storage of blockchain. Every transaction occurs between two nodes over blockchain, will notify all other nodes of the network to validate and verify the transaction to add in the block and all nodes of the network have the same copy of that transaction and it can't be repudiated because it has been added by all peers of the network [20].

There are the main components of blockchain that forms blockchain type and what is the purpose of it, include the following:

- Distributed Ledgers: ledgers are the databases of a blockchain networks and run on many participants that are engaging in the network, these databases are the same and consist of all verified transactions that happen between participants.

- Consensus Protocols: consensus protocol is an algorithm that allows securely updating the ledger when all nodes are verifying and committed the transaction through a consensus mechanism. There are many types of consensus algorithm used in blockchain like proof of work, which are used in bitcoin as an example [20].

- Encryption Methods: encryption algorithm is consisting of two keys, a public key and a private key for each participant in blockchain network. The private key is used in an authentication and encryption of sending transactions. The public key is used for decryption of receiving transactions.

- Smart Contracts: a smart contract is a software that will be run and execute over blockchain network among participants, it is containing of business logic and rules that shows the purpose of the blockchain.

- Immutable audit trail: participants can read and add transactions to a ledger, but when a transaction verifying and committed, the ledger can't be modified or deleted [5].

Blockchains use two types of cryptographic algorithms, asymmetric key algorithms, and hash functions [23]. Asymmetric cryptography is also known as digital signature because it relies on a pair of keys linked together by cryptography. It is more secure than symmetric cryptography where both sender and receiver use the same key. In blockchain all transactions are digitally signed when the sender sends a transaction, it will be encrypted by his private key and the receiver will decrypt it using a public key of the sender, this is known as asymmetric cryptography. Hashing is another type of cryptography in blockchain, as it is a process of converting any data into string of characters, hash function provides the functionality of view blockchain to every node, blockchains use the SHA 256 hashing algorithm as their hash function [23].

\section{B. Electronic Payment and Payment journey}

Electronic payment is a process of paying bills, goods, and other things online. It is processing transactions electronically via the Internet, and this process contains a lot of information describing payment details, such as payment methods and customer information. The payment journey is the process of tracking payment request between multiple components in the business channel until completion and success, as shown in Figure 1 [21].

Payment Journey Workflow Diagram

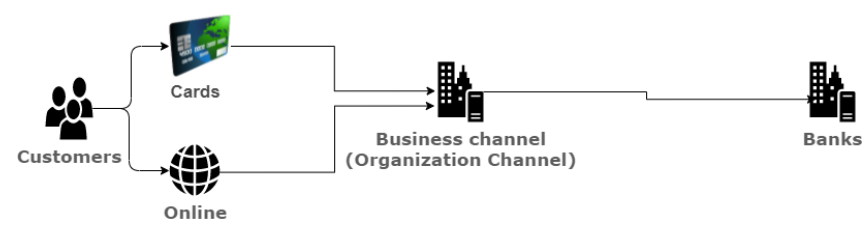

Fig. 1. Payment journey diagram.

Inside business channel, there are many components used to process and verify many different aspects of payment transactions. Each component has its configurations and a certain purpose for the payment service. These components communicate with each other with several messages, and each one has different capabilities and responsibilities. Every component has a certain shape of message for the sender action and another shape of the message for the receiver action, several transactions occurred between them aiming to achieve its target. The advantages of this cycle are being more secure and efficient for protecting sensitive data of payment transactions easy to integrate with different platforms with no trouble. The disadvantage is when any payment transaction failed it takes a long time and effort to investigate what is the problem and what is the root cause of 
it also which component has the problem, so there is need to design proposed framework using permission blockchain to overcome these problems.

\section{Related Work}

As blockchain is a promising field and still undergoing widely spread, there are much research on the blockchain with the financial industry and applying blockchain as a solution in many industries, so some of research are chosen which is little relative to this work. They had listed as following:

In this paper [7], authors describe the impact of using blockchain in financial industry, they demonstrate the items of blockchain with fintech, and show three critical challenges about using blockchain technology to be considered in upcoming work, they aim to discuss the development of blockchain for financial sector and how the financial sector can be adapted with such technology also how to manage data sharing in a more structure way. They made sixteen interviews with experts in the blockchain industry to have a good understanding of this technology. They reached out with three important propositions, the first one blockchain can be made a massive change to banks and financial services with both positive and negative impacts, and the most important thing which supports our thesis is banks and financial organizations should manage blockchain as a permission network as their data is the sensitive data and they can't afford to be in public. In addition to they need to change their understanding that blockchain is only related to Bitcoin and the cryptocurrency system. The second one is hidden knowledge, which may be a common problem in companies or organizations that adopt blockchain. The third one is to develop a comprehensive blockchain framework to manage and evaluate expert recommendations.

In the blockchain implementation study [12], author shows how to implement blockchain inside the organization by using (A-A) theory and how to help IT practitioners to implement blockchain effectively, he interviewed 24 experts as 14 from AirSouth group and ten from ChainSolution and the outcome of this study is to extend the (A-A) theory by adding experimentation phase, also give insights into how to implement blockchain in an organization as most of the other studies focus on the impact of blockchain and little is known about how to implement it. Also, the author faces some limitations like his study is based on large organizations, and his results may not be applicable to startups.

The work closest to ours but in different field (telecom) is blockchain technology in the service management domain of telecom [3], the author explores the applicability of blockchain as a solution in service management of the telecommunication industry also mentions certain challenges by employing many methods to develop a blockchain solution to implement service management then incrementally enhance that design through interviews with experts. The results of this research help various domains that planning to implement private blockchain solutions in various fields. As there is need to do in this thesis to implement private blockchain networks as a solution for this case. Also, he mentioned the benefits of applying private blockchain as immutability and transparency can resolve service management challenges as trust between different vendors in telecommunication sector also, he mentioned some technical concerns about his solution like throughput of the database that can be managed, the latency of the database, which is the time between transaction initiation and adding it in the ledger.

\section{PROPOSED FRAMEWORK}

Electronic payment is one of many shapes of digital transformation and the most promising field and has many challenges either security or performance, so the proposed framework is done to overcome the latency of tracing logs and increase the process of finding what is the root cause of the failure in the payment journey. It is found that by applying blockchain architecture as a solution for logging payment failure. The blockchain is a decentralized distributed database and real-time tracking for every transaction that happens over a blockchain network. These transactions are digitally signed, and every committed transaction cannot be modified or deleted. The Payment journey starts when the customer need to initiate payment request over the internet by using different payment methods as shown in figure 2 .

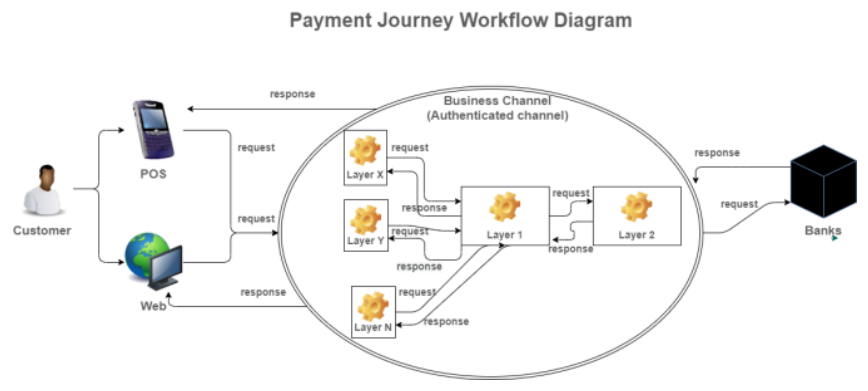

Fig. 2. Payment journey inside business channel diagram

Payment request was sent to business Channel or authenticated channel to validate and verify this request and respond at the end with success or failure of the process, the business channel consists of many layers of components that used for a certain payment method like a card or online ...etc. These components may fail the request and return with a default message to the customer. every component has a $\log$ system, and this makes tracing failures a difficult process to found which layer/component must fail and what is the root cause of the problem which invests a lot of time and effort to find what happened. Some layers may consist of small layers inside them, and the process might fail in one of them. After long research, it's found that blockchain architecture is suitable solution for tracing payment journeys and $\log$ transactions that failed with a detailed error message that helps the operation team to find out what happened, which component that transaction failed on it, what is root cause of the issue and of course, this will lead to fix these issues and make the right decision. There are types for blockchain framework like public, private (fully private, consortium), public blockchain network doesn't need permission and everyone in the network can participate in committed and verification of transactions, it is open for anyone in the entire world who can read, write and send transactions. A private network, it's a permissioned network in which there some nodes have control and govern who will join in this network and can modify or verify the transaction, a comparison is applied on which type will be suitable for the solution as shown in Table $I$.

Proceedings of $1^{\text {st }}$ International Conference on Computers and Information, ICCI 2021 
network. As shown in figure 3, the proposed framework consists of:

1) Payment Systems (Component $x$,...)

2) Blockchain Architecture:

a) blockchain network.

b) blockchain nodes.

TABLE I. BLOCKCHAIN TYPES

\begin{tabular}{|c|c|c|}
\hline Who can control & $\begin{array}{c}\text { Pome selected Nodes } \\
\text { for Consortium / one } \\
\text { node for fully private }\end{array}$ & All nodes \\
\hline $\begin{array}{c}\text { Need to be } \\
\text { internal or } \\
\text { external in } \\
\text { organization }\end{array}$ & $\begin{array}{c}\text { Most suitable for } \\
\text { internal for fully } \\
\text { private / partial } \\
\text { external for } \\
\text { consortium }\end{array}$ & $\begin{array}{c}\text { Most suitable } \\
\text { for external }\end{array}$ \\
\hline $\begin{array}{c}\text { Speed of } \\
\text { transaction }\end{array}$ & Faster & Slower \\
\hline Privacy of data & More & Less \\
\hline $\begin{array}{c}\text { Speed of real-time } \\
\text { traceability }\end{array}$ & High & $\begin{array}{c}\text { Low } \\
\text { access the } \\
\text { network } \\
\text { (permission } \\
\text { less) }\end{array}$ \\
\hline Who can access & $\begin{array}{c}\text { Only permissioned } \\
\text { nodes }\end{array}$ & $\begin{array}{c}\text { Ethereum } \\
\text { Example }\end{array}$ \\
\hline
\end{tabular}

c) smart contract (chain code)

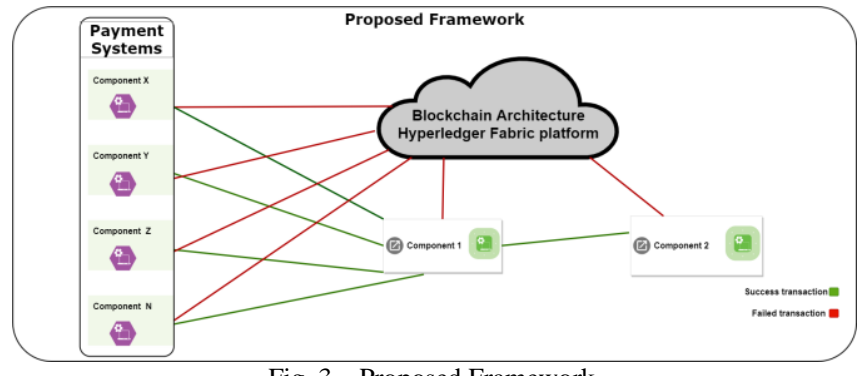

Fig. 3. Proposed Framework.

\section{A. Payment Systems}

Payment systems are group of components/layers that interact with components 1 and 2 to complete the payment process until the end. Each component has different payment method and is specified for a specific business. To be summarized, each component is an independent service, communicating with components 1 and 2 to complete the transaction. Successful transactions are shown in green, and failed transactions are shown in red.

\section{B. Blockchain architecture}

Hyperledger fabric is chosen for representing a private blockchain platform that can create a membership service provider (MSP) that controls who can access the network with cryptographic identities, ordering service which used to order the transactions and broadcast blocks to the participants over the network, endorsement, and validation policy for verifying and commit a valid transaction to the ledger. Each participant in the blockchain network represents a node which can be any machine that can be connected to the network. The Smart contract contains all business logic and rules. All participants of the network have the same smart contract to guarantees that every transaction will apply the same logic and rules. In the Hyperledger fabric, a smart contract called a chain code which does the same thing [16][20]. go through a smart contract that contains business logic and rules for the $\mathrm{BC}$ network

After reviewing several consensus algorithms, practical Byzantine fault tolerance is chosen as a consensus algorithm for the proposed solution, because PBFT provides high throughput transactions and low latency, which is the most effective advantage which is needed in the proposed framework. From the comparison, it is found that the hyperledger fabric platform is a suitable framework for building the proposed blockchain application as it depends on PBFT as consensus algorithm, and it is a permission platform, that is private and controlled by known Identities. Hyperledger fabric is an open-source enterprise-level blockchain platform. Through modular design of hyperledger, it's allowing to customize the platform itself by formulating algorithms that allow processing of identities, encryption, ordering which happen through the business 


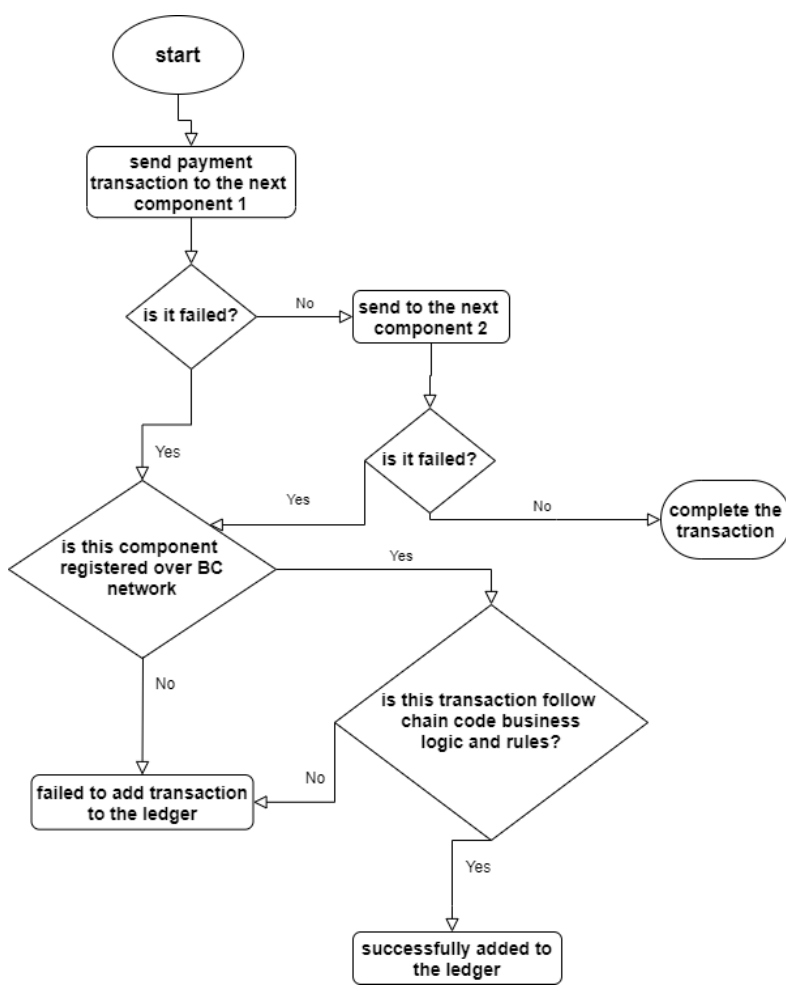

Fig. 4. Suggestion framework flowchart.

The suggestion framework flowchart as shown in figure 4, starts when any component among payment systems must send transaction to component 1If it fails, the component will interact with our blockchain network. After checking the component registered through the network, it will execute the payment $\log$ transaction and check the correctness to approve it. After that it will order the transaction via PBFT consensus algorithm, then validate transaction against implemented chain code (smart contract), finally committed and successfully added to the ledger. So all failed payment log transactions that might happened across the business organization in the blockchain network can be found, and this process will simplify the process of finding which component failed and what is the root cause of the failure. This will lead to high performance also increase the ability to fix the problem in a fast way, decreasing the time invest in every component log system and help to make the right decision.

Conclusively, Traditional Solution for troubleshooting failed payment transaction by tracking log files for every component to check where and why the trouble occurred, which not easy way at all and consumes much time, effort and resources to investigate. Furthermore, there is error margin in this troubleshooting process. However, in this research, we introduced a blockchain based troubleshooting framework. The proposed framework utilizes a permission blockchain network for logging failed payment transaction. Which it can be further queried for faster and more efficient troubleshooting. The payment transaction consists of some categories like customer information and bill details as shown in figure 5 .
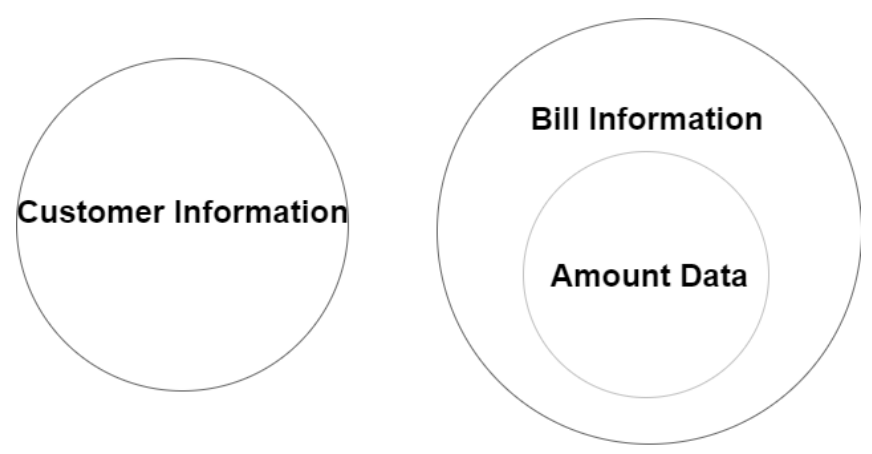

Fig. 5. Payment Transaction details.

In the proposed framework there is a format for the transaction that describes all information for failed payment transaction like sender Id, payment method, error message, transaction date, component name, Amount and log date as shown in table II.

TABLE II. TRANSACTION FORMAT

\begin{tabular}{|l|l|}
\hline Field & Description \\
\hline Sender id & $\begin{array}{l}\text { Unique identifier for } \\
\text { payment log. }\end{array}$ \\
\hline Payment method & Card or cash. \\
\hline Error message & $\begin{array}{l}\text { Error details about failed } \\
\text { payment transaction. }\end{array}$ \\
\hline Transaction date & $\begin{array}{l}\text { Date of adding the } \\
\text { transaction }\end{array}$ \\
\hline Component name & Component name. \\
\hline Amount & Amount in the transaction. \\
\hline Log date & $\begin{array}{l}\text { Date of adding transaction in } \\
\text { the ledger. }\end{array}$ \\
\hline
\end{tabular}

These fields help to identify when and where the problem happened in specific component. proposed framework uses hyperledger fabric which uses SHA 256 algorithm as encryption mechanism to encrypt every committed transaction over the blockchain network.

\section{RESULTS}

After applying the proposed framework and testing it against the current existing system as a simulation in a company working in the electronic payment field. the operation team and the development team had been interviewed to determine the time consumed to detect the problem. As a result of that, a survey consisting of 100 of incidents that happened was done, the items of this survey include the following: name of interviewee, position, the problem, and the time consumed to detect, which component affected and what is root cause of failure. By assuming the average consumed time, it is found that the average consumed time is between 15 and 30 minutes. By applying a simulation scenario from the proposed framework and compared it with the obtained data, it shows that the average of consumed time takes less than a current existing system with range between 16 and 90 seconds as shown in figure 5. 


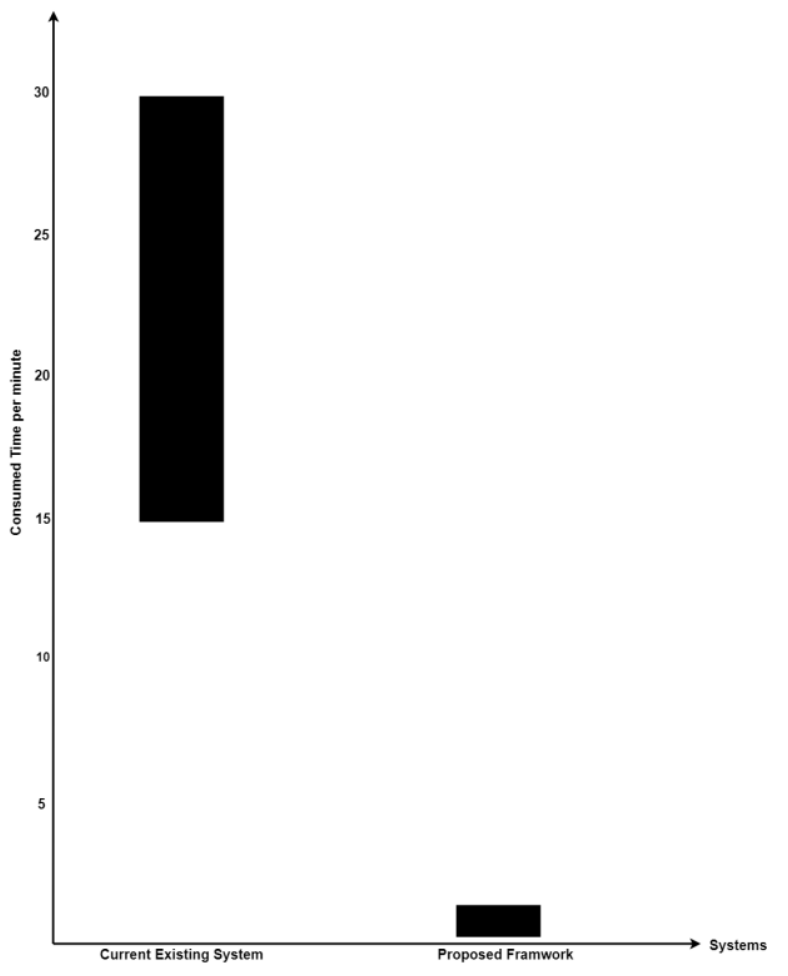

Fig. 6. Comparison between Proposed framework and the Existing system.

As a result of our research on practical byzantine fault tolerance (PBFT) consensus algorithm [19-20], it is found that this consensus algorithm provides us with the most needed advantage and highly efficient which are high throughput and low latency. Hyperledger fabric also provides us with new transaction architecture that is execution-ordervalidate. This architecture allows us to speed up the process of committed transactions and supports flexibility, scalability, and improved performance. Apply our proposed framework by using current resources, as shown in Table III.

TABLE III. RESOURCES FOR THE PROPOSED FRAMEWOEK

\begin{tabular}{|c|c|c|c|c|}
\hline & OS & $\begin{array}{c}\text { Based } \\
\text { Memory }\end{array}$ & Storage & $\begin{array}{c}\text { Virtual } \\
\text { Machine }\end{array}$ \\
\hline $\begin{array}{c}\text { Resources } \\
\text { for building } \\
\text { the proposed } \\
\text { framework }\end{array}$ & $\begin{array}{c}\text { Ubuntu } \\
\text { v20.04.2 }\end{array}$ & $8 \mathrm{~GB}$ & $40 \mathrm{~GB}$ & $\begin{array}{c}\text { Oracle } \\
\text { VM } \\
\text { VirtualBox }\end{array}$ \\
\hline
\end{tabular}

\section{Time latency}

For creating the proposed framework, hyperledger fabric v2.3 [15] has been used to build a private and permissioned blockchain network, and use go, node.js, javascript, and docker containers to implement our chain code, docker for deploying Hyperledger images. a component system has been simulated to interact with the blockchain network and the time latency was defined as it is a consumed time from submitting the transaction till it is verified and committed to the ledger as shown in figure 7 [16][22].

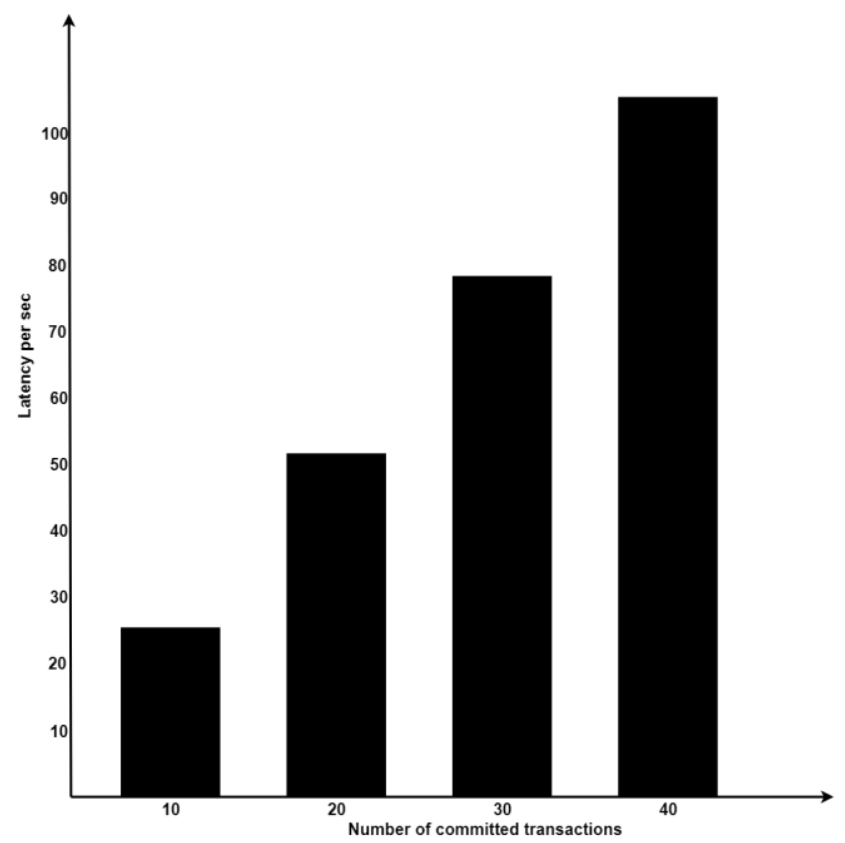

Fig. 7. Time Latency for committed transactions.

\section{A. throughput}

Throughput is defined as the number of transactions which can be transmitted per unit of time, also it is defined as the number of committed and verified transactions [22]. In hyperledger, the transaction throughput is assumed by calculating number of committed transactions / total time in seconds per committed node as shown in figure 8.

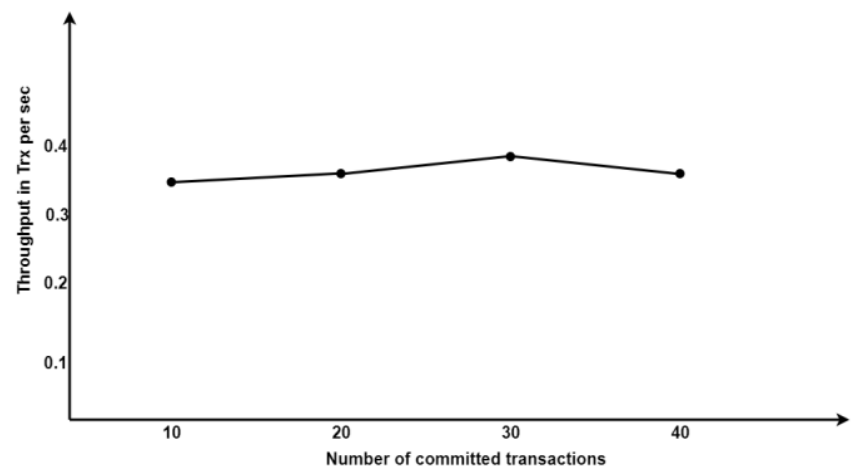

Fig. 8. Transaction throughput in the proposed framework.

The existing system using a traditional log file system, that every component add its transactions in a file system hierarchy in separated locations, which makes the process of troubleshooting the failed payment transaction more complicated and takes more time to discover what is the root cause of the problem, from the result of comparing proposed framework and existing system shows that the permission blockchain network can help to increase the process of tracing the failed payment transaction in a good way. Our results gained from the proposed framework enables us from tracing the failed transactions with little consumed time which provides more performance and efficiency. Also, by increasing the resources it is expected to decrease time latency and increase transaction throughput.

\section{IIV. CONCLUSION}


The purpose of this research is build and develop a private blockchain to track payment failure process through the payment journey to handle and trace the problems in the process. The applicability of this solution enables the organization to enhance the effectiveness and efficiency of the process of tracking payment failure, finding out what is the root cause of the problem and which component has to fail, and develop governance capabilities capitalizing on the inherent features of blockchain technology allowing:

- Use encryption technology to protect and secure transactions.

- Create a correlation between the transaction and timestamping of each transaction.

- Traceability of payment failed transactions and transaction history cannot be modified or deleted by an unauthorized node.

This provides the organization with enhanced governance over the existing domain capabilities, enables greater efficiency in the utilization of resources in electronic payment capabilities, and increasing the process of making the right decision..

\section{REFERENCES}

[1] Pilkington, M., Olleros, F. X., Zhegu, M., \&amp; Elgar, E (2015). Blockchain Technology: Principles and Applications. https://papers.ssrn.com/sol3/papers.cfm?abstract_id=2662660.

[2] Tapscott, D., \& Tapscott, A. (2017). The Impact of the Blockchain Goes Beyond Financial Services. https://hbr.org/2016/05/the-impact-of-the-blockchain-goesbeyond-financial-services

[3] Atef Ibrahim, M., Lefever, H., \&amp; De Rijk, T. (2017) APPLICABILITY OF BLOCKCHAIN TECHNOLOGY IN TELECOMMUNICATIONS SERVICE MANAGEMENT. http://liacs.leidenuniv.nl/assets/Uploads/Atef-Mohamed-nonconfidential.pdf?fbclid=IwAR10nRlGWo4nGDkHwWLKGBIqR 3xGCYqoIIeFeAhE_g5853cOylsbkHIrYr0

[4] Kareem, A., Bin Sulaiman, R., \&amp; Farooq, M. U. (2018). Algorithms and Security Concern in Blockchain Technology: A Brief Review. https://www.researchgate.net/publication/327289268_Algorithms _and_Security_Concern_in_Blockchain_Technology_A_Brief_R eview.

[5] Al-Saqqa, S., \& Almajali, S. (2020). Blockchain Technology Consensus Algorithms and Applications: A Survey. https://www.researchgate.net/publication/344227197_Blockchain _Technology_Consensus_Algorithms_and_Applications_A_Surv ey

[6] Mazonka, O. (2016). Blockchain: Simple Explanation. http://jrxv.net/x/16/chain.pdf

[7] Chang, V., P. B., H. Z., Q. X., J. Z., \& M. A. (2020). How Blockchain can impact financial services - The overview, challenges and recommendations from expert interviewees. https://doi.org/10.1016/j.techfore.2020.120166
[8] JACKSON, R. E. U. B. E. N. (2018). Scalability is Blockchain's Biggest Problem But it Can Be Resolved. https://cryptoslate.com/scalability-is-blockchains-biggestproblem-but-it-can-be-resolved/.

[9] Tsiakis, T., \&amp; Stephanides, G. (2005). The concept of security and trust in electronic payments. https://www.researchgate.net/publication/222559488_The_conce pt_of_security_and_trust_in_electronic_payments.

[10] Abdulla, A., Abubakr, A., \&amp; Ghareb, M. I. (2015). EPayment: Prospects, Challenges and Solutions in KRG Public Sector. https://www.researchgate.net/publication/281494993_EPayment_Prospects_Challenges_and_Solutions_in_KRG_Public_ Sector.

[11] Wamba, S. F., Kala Kamdjoug, J. R., Bawack, R. E., \&amp; G. Keogh, J. (2019). Bitcoin, Blockchain and Fintech: a systematic review and case studies in the supply chain. https://www.tandfonline.com/doi/full/10.1080/09537287.2019.16 31460 .

[12] (Derek) Du, W., L Pan, S., E. Leidner, D., \&amp; Ying, W. (2018). Affordances, experimentation and actualization of FinTech: A blockchain implementation study. https://www.researchgate.net/publication/328766735_Affordance S_experimentation_and_actualization_of_FinTech_A_blockchain _implementation_study.

[13] Nakamoto, S. (n.d.). Bitcoin: A Peer-to-Peer Electronic Cash System. https://bitcoin.org/bitcoin.pdf.

[14] Gao, W., G. Hatcher, W., \&amp; Yu, W. (2018). A Survey of Blockchain: Techniques, Applications, and Challenges. https://ieeexplore.ieee.org/document/8487348.

[15] Hyperledge. (n.d.). Hyperledge. https://www.hyperledger.org/use/fabric.

[16] Hyperledger Fabric. (n.d.). Hyperledger Fabric. https://hyperledger-fabric.readthedocs.io/en/latest/.

[17] Ismail, L., \&amp; Materwala, H. (2019). ArticleA Review of Blockchain Architecture and ConsensusProtocols: Use Cases, Challenges, and Solutions. https://www.mdpi.com/2073$8994 / 11 / 10 / 1198$

[18] 101 Blockchains. (2020). Permissioned Vs Permissionless Blockchains. https://101blockchains.com/permissioned-vspermissionless-blockchains/.

[19] Salimitari, M., \&amp; Chatterjee, M. (2019). A Survey on Consensus Protocols in Blockchain for IoT Networks. https://arxiv.org/pdf/1809.05613.pdf.

[20] AAX Academy. (2020). Consensus Mechanisms? How PoW, PoS, DPoS, and Other Blockchain Algorithms Work. https://academy.aaxspace.com/en/consensus-mechanisms-howpow-pos-dpos-and-other-blockchain-algorithms-work/.

[21] Kim, S. I., \& Kim, S. H. (2020). E-commerce payment model using blockchain. https://link.springer.com/article/10.1007/s12652-020-02519-5.

[22] HYPERLEDGER. (2018). Hyperledger Blockchain Performance Metrics. $\quad$ https://www.hyperledger.org/wpcontent/uploads/2018/10/HL_Whitepaper_Metrics_PDF_V1.01.p df.

[23] Sahu, M. (2021). Cryptography in Blockchain: Types \& Applications. Cryptography in Blockchain: Types \& Applications . https://www.upgrad.com/blog/cryptography-in-blockchain/. 